\title{
The challenges for molecular nutrition research 3: comparative nutrigenomics research as a basis for entering the systems level
}

\author{
Hannelore Daniel · Christian A. Drevon • \\ Ulla I. Klein · Robert Kleemann · Ben van Ommen
}

Received: 31 May 2008/Accepted: 8 September 2008/Published online: 1 October 2008

(C) Springer-Verlag 2008

\begin{abstract}
Human nutrition and metabolism may serve as the paradigm for the complex interplay of the genome with its environment. The concept of nutrigenomics now enables science with new tools and comprehensive analytical techniques to investigate this interaction at all levels of the complexity of the organism. Moreover, nutrigenomics seeks to better define the homeostatic control mechanisms, identify the de-regulation in the early phases of diet-related diseases, and attempts to assess to what extent an individual's sensitizing genotype contributes to the overall health or disease state. In a comparative approach nutrigenomics uses biological systems of increasing complexity from yeast to mammalian models to define the general rules of metabolic and genetic mechanisms in adaptations to the nutritional environment. Powerful information technology, bioinformatics and knowledge management tools as well as new mathematical
\end{abstract}

All authors are members of the European Nutrigenomics Organization (See http://www.nugo.org/author for full affiliation of all authors).

H. Daniel · U. I. Klein

Molecular Nutrition Unit, Nutrition and Food Research Center,

Technische Universität München, Freising, Germany

\section{A. Drevon}

Department of Nutrition, Institute of Basic Medical Sciences, Faculty of Medicine, University of Oslo, Oslo, Norway

e-mail: c.a.drevon@medisin.uio.no

R. Kleemann · B. van Ommen

Department of BioSciences, TNO-Quality of Life,

Leiden, The Netherlands

e-mail: ben.vanommen@tno.nl

H. Daniel ( $\square)$

Am Forum 5, 85350 Freising, Germany

e-mail:daniel@wzw.tum.de and computational approaches now make it possible to study these molecular mechanisms at the cellular, organ and whole organism level and take it on to modeling the processes in a "systems biology" approach. This review summarizes some of the concepts of a comparative approach to nutrigenomics research, identifies current lacks and proposes a concerted scientific effort to create the basis for nutritional systems biology.

Keywords Functional genomics - Comparative genetics . Systems approaches

\section{Introduction}

Mammalian metabolism has adapted to cope with rapid changes in the supply of energy, macro- and micronutrients and non-nutritional components of foods. To understand the key mechanisms underlying the metabolic regulation, the flow of information and metabolites has to be considered at all levels of the organism, starting from the molecular and cellular level onto the level of the organs' interaction. Accordingly, a systems approach aims to simulate the regulatory processes on the basis of complex experimental data sets to finally predict the outcome of any alteration or perturbation. Systems biology finally intends to describe in mathematical terms and by kinetic models most comprehensively the response of the whole organism.

Genome-wide proteome interactions are being measured and modeled at the cellular level [21] and first attempts to include and connect all "omic" layers have been reported as well $[5,18,24]$. Nutritional science has started to adopt transcriptomics $[3,20]$, proteomics $[6,8]$ metabolomics $[26,28]$ and combinations thereof [7] to assess nutritional processes in animal or human studies. Metabolic flux 
analysis $[11,12]$ and first modeling approaches on nutritional processes such as the folate cycle [15] or copper metabolism [10] have been reported. These new tools and approaches can now be used for a reassessment of known physiological and biochemical responses related to food intake and metabolic pertubations on the basis of the genome and by analysing the changes in mRNA, protein, and metabolite levels. However, for most individual nutritional components, it is not known mechanistically of how they affect body functions in the health state and even more so in view of future prevention strategies, how they may cause alterations that lead to disease. But systems approaches and pattern analysis in complex "omic" data sets can be used to define the "nutritional phenotype" on individual level, its metabolic state and its distinct reactions to altered nutritional conditions.

As most principles of biology apply to all living systems and all living systems rely on the supply of nutrients and respond to their nutritional environment, there is a dimension of nutrigenomics research that one may call "general nutrigenomics principles". This means that it should seek to identify and compare metabolic and regulatory processes essentially independent of the organism and its specific genetic make up. Fitting to the "life's complexity pyramid" [17], organism specificity increases as closer we get to individual genomes and genes, whereas the universality of the processes emerges as we move away from the genome or genes. Comparative nutrigenomics may serve as a guideline in exploring to which extent common biological principles or organism-specific processes can be detected in systems biology approaches to nutrition.

Comparative nutrigenomics needs to identify and explore the fundamental principles underlying the organization and the evolution of biological systems in the context of the quantity and quality of nutrient supply. What are the common mechanisms and design principles and what have been the selection pressures of evolution that caused these processes to develop? Evolution is inherently the continuous adaptation to a changing environment, including a varying availability of energy and nutrients, and this is achieved by modularity in the responses. Modularity is a prime principle in the organization of prokaryotic genomes which have transcriptional unitsoperons-that translate into multi-protein functional units. Modularity is also inherent in the organization of regulatory and metabolic networks in higher organisms. However, methods for identification of these modules are still under development and in nutritional research, no systematic explorations has been undertaken so far. Yet, all the knowledge gathered over decades on metabolism, physiology, and biochemistry and on the underlying regulatory processes may serve to define the "general rules of metabolic control". They have to be separated from the more specialized mechanisms that emerged later in evolution when organisms diverged to creatures with organs of specialized functions and superordinate hormonal and neuronal regulatory networks that all need to be synchronized with the cellular effects of the nutrients.

One of the most intriguing questions is whether living in a given nutritional environment conditions the genome and predisposes it to better or faster adjustment to a new or similar nutritional situation later in life-time [23]. Epigenetic effects, mainly by modifications of gene sequence accessibility, can be imprinted prenatally and shape the protein expression pattern. This was first shown in mice where altered methylation of gene promoters induced in the F1 generation by protein restriction during fetal development was inherited by the F2-generation [4]. Such a nongenomic transmission of environmental information might have originally evolved to improve the chances of the offspring to survive under the conditions experienced by the mother and predicted for the next future. However, today nutritional conditions can change rapidly, e.g., by emigration or by socioeconomic changes, and then the epigenetic response will "mismatch" the environment experienced throughout life and cause increasing risks for diseases [23]. It is still not known how this "cellular or genomic memory" works and how the facts and factors of the nutritional environment are transmitted into the genome to cause sustained and long-term changes in the organism.

\section{The goals of comparative nutrigenomics}

The prime goal of comparative nutrigenomics studies is to identify the modular architecture controlling nutritional processes. This includes the analysis of the effects that cause a system to drive away from equilibrium into instabilities and the reactions needed that allow the system to reach a new steady state of metabolic adaptation. We need to define the time-scales of these adaptations and their extent to which they feed-back into the genome to cause a "memory-like" alteration. As the interaction between the components in a cellular system usually extend only over a short range, a critical state is needed to propagate a pattern that goes all the way across the organism to see major changes in the global organization in which details of the particular system get obliterated. Such a "critical state" may be defined in terms of nutrition as a severe state of starvation, a micronutrient deficiency or diets providing extreme nutrient compositions. To be able to assess the global organization in a biological system models of increasing complexity are needed-from single cells to multi-cellular simple organisms to mammalian (rodent) models and finally humans. 
Comparative nutrigenomics should be the basic science approach that helps to define the principles of metabolic and genetic adaptations of mammals to changes in the nutritional environment. Because studies in humans are limited due to ethics and sample availability, only model systems provide the chance to deeper explore the fundamental rules and modules by enabling high throughput and thus using the power of comparative nutrigenomics approaches. When organisms are lined up by increasing genetic, morphological and functional complexity and in parallel by increasing complexity of their nutritional environment and needs, they exhibit decreasing applicability, feasibility, and costs for the use in functional genomics techniques. The advantages and disadvantages of the different models of increasing complexity in view of cell populations and tissues and nutritional needs are depicted in Fig. 1.

\section{Yeast}

Yeast cells are the best studied simple eukaryotic model for systems biology [14]. Yet, they lack cellular specialization and have a less complex interplay of extracellular (hormonal and neuronal inputs) and intracellular signaling processes controlling metabolic adaptation than multi-cellular organisms. However, more basic processes such as the prime metabolic pathways provide valuable modules that can be translated into mammalian cell metabolism. Thus, the challenge is to make optimal use of the vast repertoire of knowledge, tools, and transgenic models available for yeast and translate these results into mammalian systems biology.

\section{Invertebrates such as Caenorhabditis elegans}

In comparison to yeast, $C$. elegans is a complex organism (around 1,000 cells) with a variety of cell types and with essentially all sensing and signaling pathways in place as in higher vertebrates (for example the insulin signaling cascade). C. elegans can be genetically modified like almost no other multicellular system (knock-outs, multiple knockouts, thousands of mutant lines, numerous reporter lines, and RNAi for every gene easy to perform). It has fast reproduction, growth and development and "omics" technologies can be applied like in all other systems. The drawback is that $C$. elegans does not have blood and therefore not a central distribution system for a complex inter-organ metabolism. However, it is the "fastest" system available if it comes to a certain level of complexity allowing high-throughput analyses [9]. Dietary studies, however, are more difficult to conduct because little is known about metabolism in worms and food is normally living E. coli. Drosophila melanogaster is also a suitable model with similar advantages and disadvantages as worms and has provided major contributions to basic science questions that relate to nutrition [22].
Fig. 1 The comparative nutrigenomics approach employing various models systems of increasing complexity with their advantages and disadvantages

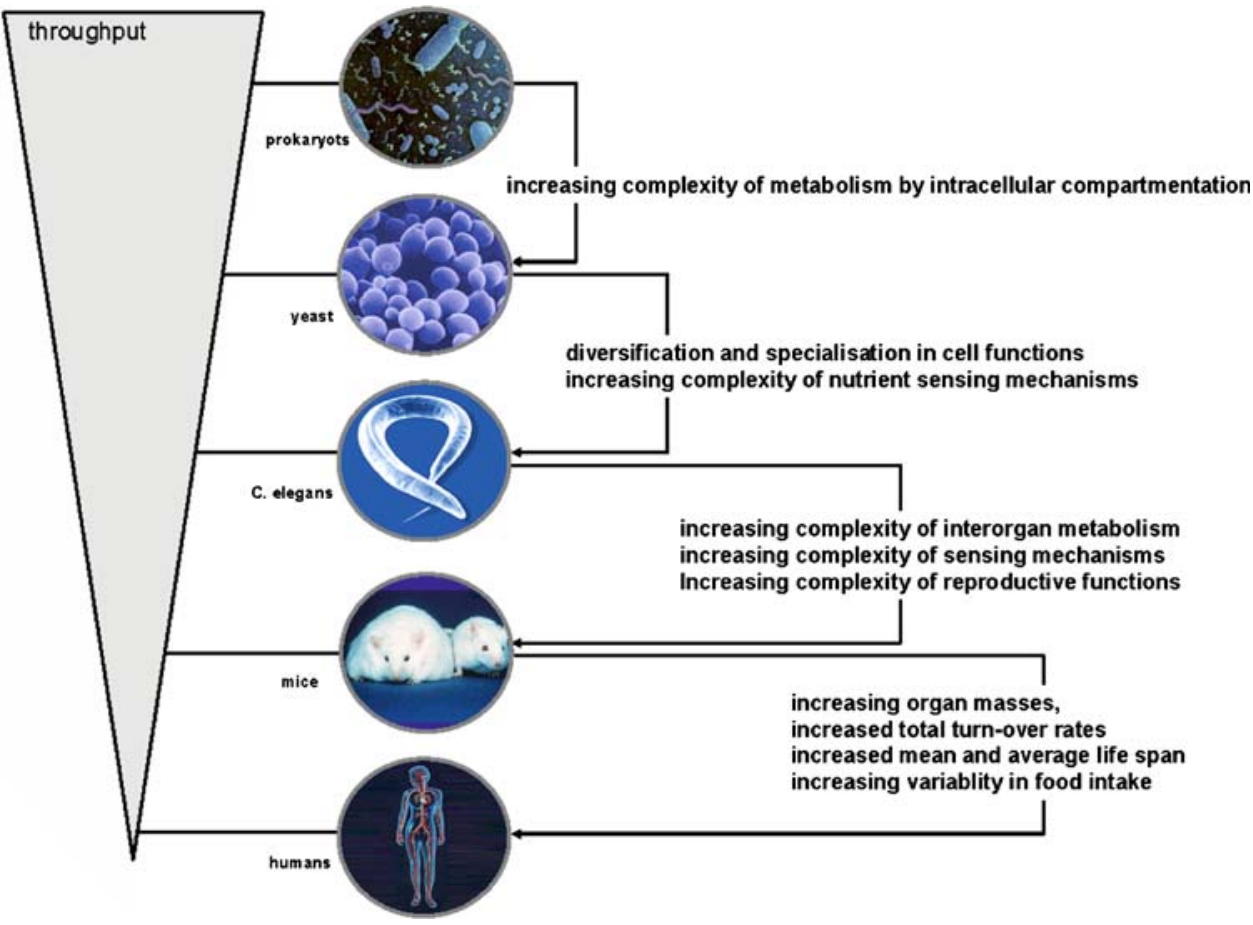




\section{Rodents}

Rats are the traditional animal model in nutrition research with superior qualities in handling and conducting in vivo studies. Consequently data from rat studies give a larger database than those for mice. Rats are also more complex in feeding behavior and food choices and will eat a wider variety of foods, in different forms, than mice. However, the lack of significant numbers of mutant strains is a limitation. Mice have only recently become the prime mammalian model species by the ease to create transgenic lines with gene-deletions including tissue- or cell-specific inactivation or targeted over-expression of mouse or human genes in different genetic backgrounds. The numerous transgenic or mutant mouse lines available allow mammalian monogenetic diseases to be studied [16] or even complex traits like aging in combination with advanced genetics and the ease of fast breeding and line propagation [2]. Transgenic mice models also give access to nutritional studies employing transcriptome, proteome, and metabolome analysis. Mice models are the choice to assess the plasticity and connectivity of metabolism at a whole and at the level of responses in specialized organs and the inter-organ relationship. There are numerous transgenic lines with knock-outs and knock-ins as well as a battery of reporter lines for gene expression analysis based on expression of the green fluorescent protein (GFP) or related proteins or luciferase-based assay systems. All "omics" can be applied-but throughput is somehow limited in terms of the organ sizes and the amount of biosamples needed for analysis. Although mice would be a reasonable model for generational studies on nutritional effects, these studies need long-term support and a huge infrastructure and are therefore difficult to conduct.

\section{The challenges}

Some of the major current deficiencies (besides the technological limitations in the different "omics"-approaches, which are dealt with in a separate review in this series) are listed below:

- Lack of well-defined nutritional intervention studies that allow the modular character of the responses to be studied and recognized. The more complex an organisms is, the more difficult this is and the lower is the throughput.

- Lack of appropriate experiments in which organisms of increasing complexity are exposed to the same dietary maneuvers for assessing whether there are common patterns (modules) in the responses.
- Lack of knowledge on the importance of imprinting/ epigenetic effects on metabolic adaptation.

- Lack of any systematic study that addresses "genetic preconditioning" to the same challenge in the same organism over numerous generations (evolutionary nutrigenomics).

- Lack of knowledge on the functional role of single nucleotide polymorphism (SNP); so far mainly identified by association studies in their cellular or systemic setting.

These challenges may be met by performing specific studies, of which a number of examples are:

- Experimentally define the advantages and limitations of the various model systems available.

- Provide a "proof of principle" study demonstrating that there are general rules and modular responses in organisms adaptation to changes in the nutritional environment at all levels of complexity (systems approach).

- Explore to which extent and how the diet causes a "preconditioning" of the genome by repeated challenges (over generations) with the same diet.

- Explore the plasticity of metabolic adaptations ("critical state" approach) in animal models and then take it into a human study.

- Define a dietary challenge experiment (iso-energetic, low versus high carbohydrate/protein) as "proof of principle" and study the response with all "omics" as a function of time in yeast, $C$. elegans and mouse lines (in different organs and plasma, urine), and derive patterns of responses (between organs, between organisms), and try to identify modules.

- Define a set of feeding experiments using a variety of diets (alterations in macronutrient composition) to study the plasticity of responses (threshold approach) in C. elegans (fastest system) and mice lines (most complex systems).

- Define an "evolutionary nutrigenomics" study with a repeated challenge to the same dietary maneuver in the same model (line) over various generations (mimic evolution in the laboratory) in C. elegans (fast model) and a mouse line (slow model) and try to identify the underlying mechanisms of "genetic adaptation" such as epigenetic alterations, mutations etc.

- Design a "proof of principle" study in genetically welldefined cohorts with the "critical state" approach to assess the plasticity of metabolic adaptation.

- Define a nutritional intervention study in genetically well-defined groups (twins or SNP-defined cohort) for the determination of plasticity of metabolic responses and "proof of principle" of modularity of adaptation. 


\section{Examples of the power of comparative genomics approaches}

Although the examples found in the literature do not deal with nutrition in the first place, they may serve as paradigms for the principles in comparative genetics and genomics approaches across species. Initially, comparative genomics was targeted toward defining the synteny of genes between species. As more and more genomes are defined that span now a wide range or organisms in the evolutionary tree more options arise and more tools are available to link also comparative genetics with comparative biology. Comparative genomics approaches take advantage of all the functional genetic information of all organisms to study human physiology and diseases, for identifying gene functions and regulatory principles.

\section{Example: longevity}

Aging research mainly in invertebrate model organisms has led to the identification of many genes that influence life span but only a few of these genes have been examined in the context of mammalian aging. Despite the fact that the mechanisms underlying longevity are not fully elucidated, it is known that mutation in genes that share similarities with those in humans involved in the insulin/insulin-like growth factor I (IGF-I) signaling pathway can significantly extend life span in yeast, worms, fruit flies, and rodents [29]. This suggests that the fundamental mechanisms for lifespan determination are evolutionarily conserved from yeast to mammals.

Long-living mutant lines-whether worms or miceshare some important phenotypic characteristics including an increased insulin sensitivity as well as reduced IGF-I plasma levels. Recent findings also provide intriguing evidence for the involvement of insulin and IGF-I in the control of aging and longevity in humans [27]. There seems to be a unifying mechanism in the genetics of longevity.

With a comparative functional genomics approach using the yeast Saccharomyces cerevisiae and the nematode $C$. elegans, recently a first quantitative analysis of the conservation of longevity genes was performed [25]. Based on worms with gene-deletions and known life span alterations, single gene-deletions of the yeast orthologs were produced with similar changes in replicative life span suggesting that genes that affect aging processes have been conserved during evolution not only in sequence, but also in function. Among the gene pairs that provided longevity in yeast and worms mainly those involved in nutrient sensing and metabolic processing as well as protein translation control could be identified. Similar overrepresented gene groups encoding proteins involved in energy production and proton transport have been identified in various screens with
D. melanogaster and C. elegans [13] and by comparative gene expression profiling in young and old flies (head and thorax tissue). In extension of these approaches a recent study employing transcript profiling and comparing 81 muscle samples from humans of varying ages identified 250 age-regulated transcripts [30, 31]. Those could be grouped into six genetic pathways of which four pathways displayed increased expression with age (extracellular matrix, cell growth, complement activation, and cytosolic ribosome), while two pathways showed decreased expression with age and here genes encoding subunits of the mitochondrial electron transport chain were again overrepresented. When those human data were compared with age-dependent transcript profiles from worms, flies and mice a similar age-dependent regulation in all four species was identified as a common "aging signature". A dedicated website of the University of Southern California called Gene Aging Nexus (http://gan.usc.edu) is an useful resource for this comparative analysis of age-dependent transcript profiles. It contains currently 74 data sets obtained from six species (yeast, worms, flies, mice, rat, and human) that allows differential gene expression and co-expression analysis with an embedded annotation module [19].

These examples of comparative genomics to define the processes of aging have revealed a common pattern of genes-independent of the species - that play a crucial role in organisms life span and particular the electron transport chain proteins that decrease on average twofold in expression with age in all organisms could prove to be the best pool for identification of robust biomarkers of aging. That the respiratory chain is found in the center of these seemingly species-independent aging processes may be no surprise since the evolutionary pressure of nutrient availability and thus substrate pools for mitochondrial respiration was most crucial for reproductive life span and a new generation of organisms. A need for longevity per se seems not to have been a profound or important evolutionary pressure.

Example: immunity

Another important selective pressure in evolution that may have shaped genomes in terms of common principles has been to withstand infections. In the organismic defence against viruses, bacteria, or nematodes innate immunity plays a prominent role. An interesting approach to identify regulators of innate immunity as common genes that contribute to the expression of immune defence processes a comparative screen of $C$. elegans and mouse macrophages was performed [1]. Genes that altered innate immune responsiveness in $C$. elegans were validated in murine macrophages and that let to the discovery of 11 genes that 
regulate the innate immune response in both systems and the subsequent identification of a protein interaction network with a conserved role in innate immunity regulation.

\section{Conclusion}

The examples demonstrate that comparative genomics leads to gene signatures and corresponding protein families or even networks that are represented in simple as well as in highly developed organisms and which one may call "signatures of the principle of life". In this respect it can be expected that comparative nutrigenomics will similarly reveal a large number of similar networks because all organisms-despite the fact that they do not all need the same nutrients-they certainly rely on the supply of some common nutrients for purposes of energy production, cell and body maintenance and reproductive functions. And to better understand these principles of life driven by nutrition and highly dependent on nutrition will ultimately lead to Nutritional Systems Biology.

\section{References}

1. Alper S, Laws R, Lackford B et al (2008) Identification of innate immunity genes and pathways using a comparative genomics approach. Proc Natl Acad Sci USA 105:7016-7021

2. Bartke A (2008) New findings in gene knockout, mutant and transgenic mice. Exp Gerontol 43:11-14

3. Bouwens M, Afman LA, Müller M (2007) Fasting induces changes in peripheral blood mononuclear cell gene expression profiles related to increases in fatty acid beta-oxidation: functional role of peroxisome proliferator activated receptor alpha in human peripheral blood mononuclear cells. Am J Clin Nutr 86:1515-1523

4. Burdge G, Slater-Jefferies J, Torrens C (2007) Dietary protein restriction of pregnant rats in the $F_{0}$ generation induces altered methylation of hepatic genes promoters in the adult male offsprings in the $F_{1}$ and $F_{2}$ generation. Br J Nutr 97:435-439

5. Clish CB, Davidov E, Oresic M et al (2004) Integrative biological analysis of the APOE*3-leiden transgenic mouse. OMICS 8: 3-13

6. de Roos B, Rucklidge G, Reid M et al (2005) Divergent mechanisms of cis9, trans11- and trans10, cis12-conjugated linoleic acid affecting insulin resistance and inflammation in apolipoprotein E knockout mice: a proteomics approach. FASEB J 19:1746-1748

7. Frey IM, Rubio-Aliaga I, Siewert A et al (2007) Profiling at mRNA, protein, and metabolite levels reveals alterations in renal amino acid handling and glutathione metabolism in kidney tissue of Pept2-/- mice. Physiol Genomics 28:301-310

8. Fuchs D, Vafeiadou K, Hall WL et al (2007) Proteomic biomarkers of peripheral blood mononuclear cells obtained from postmenopausal women undergoing an intervention with soy isoflavones. Am J Clin Nutr 86:1369-1375

9. Gunsalus KC (2008) A Caenorhabditis elegans genetic-interaction map wiggles into view. J Biol 7:8
10. Harvey LJ, Dainty JR, Hollands WJ et al (2005) Use of mathematical modeling to study copper metabolism in humans. Am J Clin Nutr 81:807-813

11. Hellerstein MK, Murphy E (2004) Stable isotope-mass spectrometric measurements of molecular fluxes in vivo: emerging applications in drug development. Curr Opin Mol Ther 6:249264

12. Lee WN, Go VL (2005) Nutrient-gene interaction: tracer-based metabolomics. J Nutr 135:3027S-3032S

13. McCarroll SA, Murphy CT, Zou S et al (2004) Comparing genomic expression patterns across species identifies shared transcriptional profile in aging. Nat Genet 36:197-204

14. Mustacchi R, Hohmann S, Nielsen J (2006) Yeast systems biology to unravel the network of life. Yeast 23:227-238

15. Nijhout HF, Reed MC, Budu P, Ulrich CM (2004) A mathematical model of the folate cycle: new insights into folate homeostasis. J Biol Chem 279:55008-55016

16. Oliver PL, Bitoun E, Davies KE (2007) Comparative genetic analysis: the utility of mouse genetic systems for studying human monogenic disease. Mamm Genome 18:412-424

17. Oltvai ZN, Barabási AL (2002) Systems biology life's complexity pyramid. Science 298:763-764

18. Oresic M, Clish CB, Davidov EJ et al (2004) Phenotype characterisation using integrated gene transcript, protein and metabolite profiling. Appl Bioinformatics 3:205-217

19. Pan F, Chiu CH, Pulapura S et al (2007) Gene aging nexus: a web database and data mining platform for microarray data on aging. Nucleic Acids Res 35(Database issue):D756-D759

20. Rakhshandehroo M, Sanderson LM, Matilainen M et al (2007) Comprehensive analysis of PPARalpha-dependent regulation of hepatic lipid metabolism by expression profiling. PPAR Res 2007:26839

21. Rual JF, Venkatesan K, Hao T et al (2005) Towards a proteomescale map of the human protein-protein interaction network. Nature 437:1173-1178

22. Ruden DM, Lu X (2006) Evolutionary conservation of metabolism explains how Drosophila nutrigenomics can help us understand human nutrigenomics. Genes Nutr 1:75-84

23. Scarino ML (2008) A sideways glance. Do you remember your grandmother's food? How epigenetic changes transmit consequences of nutritional exposures from one generation to the next. Genes Nutr 3:1-3

24. Schnackenberg LK, Jones RC, Thyparambil S et al (2006) An integrated study of acute effects of valproic acid in the liver using metabonomics, proteomics, and transcriptomics platforms. OMICS 10:1-14

25. Smith ED, Tsuchiya M, Fox LA et al (2008) Quantitative evidence for conserved longevity pathways between divergent eukaryotic species. Genome Res 18:564-570

26. Stella C, Beckwith-Hall B, Cloarec O et al (2006) Susceptibility of human metabolic phenotypes to dietary modulation. J Proteome Res 5:2780-2788

27. Suh Y, Atzmon G, Cho MO et al (2008) Functionally significant insulin-like growth factor I receptor mutations in centenarians. Proc Natl Acad Sci USA 105:3438-3442

28. Walsh MC, Brennan L, Pujos-Guillot E et al (2007) Influence of acute phytochemical intake on human urinary metabolomic profiles. Am J Clin Nutr 86:1687-1693

29. Wenzel U (2006) Nutrition, sirtuins and aging. Genes Nutr $1: 85-94$

30. Zahn JM, Sonu R, Vogel H et al (2006) Transcriptional profiling of aging in human muscle reveals a common aging signature. PLoS Genet 2:e115

31. Zahn JM, Kim SK (2007) Systems biology of aging in four species. Curr Opin Biotechnol 18:355-359 